\title{
Investigation of Water Mobility and Diffusivity in Hydrating Micronized Low-substituted Hydroxypropyl Cellulose, Hydroxypropylmethyl Cellulose, and Hydroxypropyl Cellulose Matrix Tablets by Magnetic Resonance Imaging (MRI) ${ }^{1)}$
}

\author{
Masazumi KoJIMA* and Hiroaki NAKAGAMI \\ Tokyo Pharmaceutical Research Center, Pharmaceutical Technology Research Laboratories, Daiichi Pharmaceutical Co., \\ Ltd.; 1-16-13 Kita-Kasai, Edogawa-ku, Tokyo 134-8630, Japan. \\ Received August 9, 2002; accepted September 18, 2002; published online September 19, 2002
}

The water mobility and diffusivity in the gel-layer of hydrating low-substituted hydroxypropyl cellulose (LH41) tablets with or without a drug were investigated by magnetic resonance imaging (MRI) and compared with those properties in the gel-layer of hydroxypropylmethyl cellulose (HPMC) and hydroxypropyl cellulose (HPC) tablets. For this purpose, a localized image-analysis method was newly developed, and the spin-spin relaxation time $\left(T_{2}\right)$ and apparent self-diffusion coefficient (ADC) of water in the gel-layer were visualized in one-dimensional maps. Those maps showed that the extent of gel-layer growth in the tablets was in the order of HPC $>$ HPMC $\gg$ LH41, and there was a water mobility gradient across the gel-layers of all three tablet formulations. The $T_{2}$ and ADC in the outer parts of the gel-layers were close to those of free water. In contrast, these values in the inner parts of the gel-layer decreased progressively; suggesting that the water mobility and diffusivity around the core interface were highly restricted. Furthermore, the correlation between the $T_{2}$ of ${ }^{1} \mathrm{H}$ proton in the gel-layer of the tablets and the drug release rate from the tablets was observed.

Key words magnetic resonance imaging; hydroxypropyl cellulose; spin-spin relaxation time; diffusion; micronized low-substituted hydroxypropylcellulose; hydroxypropylmethyl cellulose

Hydrogel-forming polymers such as hydroxypropylmethyl cellulose (HPMC) and hydroxypropyl cellulose (HPC) have been widely used for controlled release matrix dosage forms. ${ }^{2,3)}$ We previously reported that micronized low-substituted hydroxypropyl cellulose (LH41) has a potential use as a directly compressible carrier for a controlled release tablet. ${ }^{4,5}$ Kawashima et al. ${ }^{6}$ also reported that micronized LH41 works as a controlled release matrix. In our previous research, ${ }^{7)}$ we reported that the magnetic resonance imaging (MRI) method was a useful tool for evaluating the swelling property and the water mobility in hydrating matrix tablets. It was found that LH41 swelled much less than did HPC and HPMC, and that water mobility is highly restricted in all three tablet formulations.

The present paper extends our previous work to a study of the localized distribution of spin-spin relaxation time $\left(T_{2}\right)$ and apparent self-diffusion coefficient (ADC) of the water in the gel-layer of the hydrating LH41 tablets in order to understand the role of water in drug release from hydrophilic matrix tablets. For this purpose, a localized image-analysis method was newly developed, and one-dimensional maps of $T_{2}$ and ADC across the gel-layer were illustrated. Furthermore, the correlation between the $T_{2}$ of ${ }^{1} \mathrm{H}$ proton in the gellayer of the tablets and the drug release rate from the tablets was evaluated.

\section{Experimental}

Materials Theophylline anhydrous (Shiratori Seiyaku Co., Ltd., Japan) and procaine amide hydrochloride (Daiichi Pure Chemical Co., Ltd., Japan) were selected as a slightly water-soluble drug and a very water-soluble drug, respectively. They were each finely pulverized with a sample mill (KII-1, Fuji Electronic Industry Co., Ltd., Japan) under the following conditions: number of hammers, six; and size of screen openings, $0.5 \mathrm{~mm}$. Their mean volume particle sizes were about $10 \mu \mathrm{m}$, as measured with a laser-diffraction particle analyzer. Micronized low-substituted hydroxypropyl cellulose (LHPC) (type LH41, mean particle size, about $5 \mu \mathrm{m}$, Shin-Etsu Chemical Co.,
Ltd., Japan), HPC (type HPC-H, particle size, under $150 \mu \mathrm{m}$, Nippon Soda Co., Ltd., Japan), and HPMC (type Metholose 60SH-4000, Shin-Etsu Chemical Co., Ltd., Japan) were generously donated by the respective manufacturers.

Matrix Tablet Preparation Each of polymer and model drug, in the ratio of $2: 1$ or $3: 1$ by weight, were blended in a diffusion mixer for $10 \mathrm{~min}$. The mixture of 300 or $400 \mathrm{mg}$, corresponding to $100 \mathrm{mg}$ of the drug, was directly compressed into tablets with a universal testing machine (Autograph, type AG-5000B, Shimadzu Co., Ltd., Japan) under the following conditions: compression force, $400 \mathrm{kgf}$; compression speed, $10 \mathrm{~mm} / \mathrm{min}$; punches, flatface- $10 \mathrm{~mm}$ in diameter. The plain polymer weighing $500 \mathrm{mg}$ was also compressed under the same conditions.

NMR Imaging The experiments were performed on a Bruker BIOSPEC CSI 47/40 NMR spectrometer equipped with a $4.7 \mathrm{~T}$ superconducting magnet system operating at $200 \mathrm{MHz}$ for ${ }^{1} \mathrm{H}$-NMR. Images were taken at room temperature.

$T_{2}$-weighted images were produced in the standard manner by using multiple spin echo (MSE) methods ${ }^{8)}$ and the imaging parameters were as follows: recovery time (TR), $14000 \mathrm{~ms}$; echo time (TE), from 20 to $240 \mathrm{~ms}$; two averages; $256 \times 256$ lines; slice thickness, $5 \mathrm{~mm}$.

The ADC of water was measured based on the method established by Stejskal and Tanner. ${ }^{9)}$ In fact, the diffusion-weighted images were produced by inserting gradient pulses on either side of the $180^{\circ}$ refocusing pulse. The gradient factor $b$ was obtained as the strength of the gradient pulse applied in one direction, and it was changed from 0 to $80000 \mathrm{~s} / \mathrm{cm}^{2}$.

One-Dimensional Mapping Methods for the $T_{2}$ and ADC of Water across Matrix Tablets The flow diagram for mapping of $T_{2}$ and ADC is shown in Fig. 1. The sample was hydrated in a glass bottle filled with $15 \mathrm{ml}$ of ultrapure water replaced with nitrogen gas. The sample was then placed inside the core of the MRI for imaging. Images were taken from the coronal planes through the center of the tablet oriented as shown in Fig. 1a. A $T_{2}$ weighted image of the tablet in the bottle filled with purified water was taken, and a typical $T_{2}$-weighted image as shown in Fig. $1 \mathrm{~b}$ was acquired. The outermost circle in Fig. 1b represents the glass bottle, the inner moiety which appears clear and white is the aqueous phase, the innermost black moiety is the glassy core, and the moiety between the aqueous phase and the glassy core indicates the gel-layer, which increased during the hydration process. These $T_{2}$-weighted images were then converted into the bitmapped images as shown in Fig. 1c. The signal intensities of each pixel along the white line marked across the bitmapped images were used for the analyses; they were digitalized to numerical values, and divided by 256 with an image 


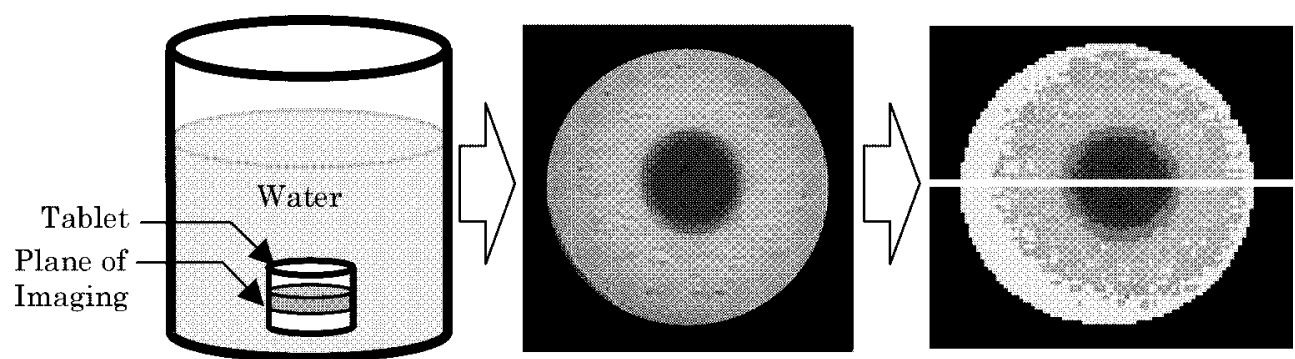

(a) Plane of the Image through the

(b) Typical $T_{2}$-weighted

(c) Bitmapped Image. Sample. Image.
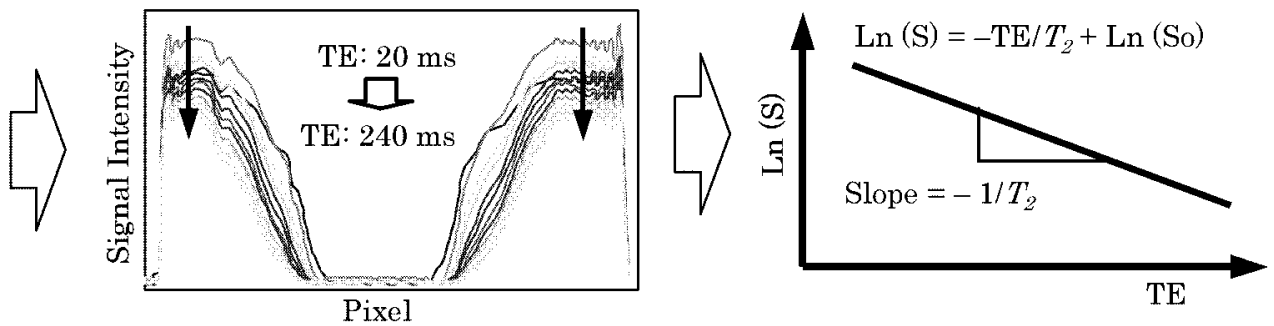

Fig. 1. Analytical Methods for Calculating the Localized $T_{2}$ within the Hydrating Tablets

analysis apparatus (Quantimet 600, Leica, Japan). The interval of 1 pixel corresponds to $0.3125 \mathrm{~mm}$.

The spin-spin relaxation time $\left(T_{2}\right)$ of water in the hydrated tablets was calculated as follows. The signal intensity of each pixel of the $T_{2}$-weighted image was measured. The $T_{2}$ is given by:

$$
S=S_{0} \exp \left(-\mathrm{TE} / T_{2}\right)
$$

where $S$ is the signal intensity after TE, and $S_{0}$ is the unattenuated signal intensity. A plot of the naturalized logarithm of $S$ of each pixel versus TE should be a straight line with a slope of $-1 / T_{2}$.

The ADC of water in the hydrated tablets was calculated as follows. The signal intensity $(S)$ of each pixel of the diffusion-weighted images was measured. The ADC is given by:

$$
S=S_{0} \exp \left\{-\operatorname{ADC}\left(b-b_{0}\right)\right\}
$$

where $b$ is the gradient factor, and $S_{0}$ and $b_{0}$ are $S$ and $b$ respectively without inserting gradient pulse. A plot of $\operatorname{Ln}\left(S / S_{0}\right)$ versus $\left(b-b_{0}\right)$ should be a straight line with a slope of $-\mathrm{ADC}$.

Assay for Theophylline and Procaine Amide Hydrochloride To examine the relationship between the $T_{2}$ and drug release, the amount of drug released from the tablet into a glass bottle filled with $15 \mathrm{ml}$ of ultrapure water was measured after $20 \mathrm{~h}$ hydration. Theophylline and procaine amide hydrochloride were assayed spectro-photometrically at 272 and $278 \mathrm{~nm}$, respectively.

\section{Results and Discussion}

One-Dimensional $T_{2}$ and ADC Maps of the Various Tablets without Drug The $T_{2}$-weighted and diffusionweighted images taken through the coronal plane of the three kinds of hydrated tablets prepared from LH41, HPMC, and HPC alone, were produced by using MRI. As reported previously, ${ }^{7}$ it was observed through the images that all tablets were hydrated as shown in Fig. 1b. That is, the glassy core could be seen as a black moiety, and the gel-layer expanded around the core as well as the aqueous phase, which clearly looks white at the outermost moiety.

Figure 2 shows the one-dimensional maps of the $T_{2}$ and ADC values across the tablets immersed for $20 \mathrm{~h}$ in water. In this fig., pixels 0 and 60 of the $\mathrm{x}$-axis indicate the center of the tablet core and the outermost aqueous phase in the glass bottle, respectively, the dashed line (pixel 16) indicates the original position of interface between tablet and water.

In the case of the hydrated LH41 tablet, the moiety of the glassy core, gel-layer, and aqueous phase may be determined by the $T_{2}$ relaxation time; whose range for each part corresponds to pixels 0 to 26 , pixels 26 to 31 , and pixels 31 to 60 respectively in the $T_{2}$ map. The one-dimensional $T_{2}$ map shows that the position of the original core interface had moved to the outside to pixel 26, and that the $T_{2}$ value of the water around the core was very short compared with that in the outer aqueous phase. The $T_{2}$ values in the region of the gel-layer (pixels 26 to 31) increased from the inner side to the outer side of the tablet and reached a constant-limiting value in the region of the aqueous phase. The $T_{2}$ values could not be obtained for the range of pixels 0 to 26 , because there was not enough water in the swelling core to detect the intensity of the NMR signal.

On the other hand, for the hydrated HPMC and HPC tablets, the moieties of the glassy core, gel-layer, and aqueous phase correspond to the pixels in the ranges of 0 to 12 , 12 to 42 , and 42 to 60 for the hydrated HPMC tablets, and in the ranges of 0 to 14,14 to 46 , and 46 to 60 for the hydrated HPC tablets, respectively, as seen in the $T_{2}$ maps. The $T_{2}$ maps of these tablets reveal that the positions of the core/gel interface have moved to the inside, compared with the original position of the tablet interface, and that the gel/water interface moved remarkably toward the outside of the tablet. The $T_{2}$ values of the water around the core were very short; but the $T_{2}$ values in the region of the gel-layer gradually increased with the distance from the core interface, up to the constant-limiting value in the region of the aqueous phase. These results indicate that the hydrated HPMC and HPC tablets swelled and formed a wide gel-layer. The extent of the gel-layer growth was almost equal for the HPMC and HPC tablets; and the water mobility in the wide gel-layer was strongly restricted, the same as in the thin gel-layer of the LH41 tablet.

These results indicate that the hydrated tablet consisting of LH41 which is a water-insoluble polymer, swelled to a much less extent compared with the hydrated tablets consisting of HPC and HPMC which are water-soluble polymers, and formed a thin gel-layer at the core interface. The water in the 

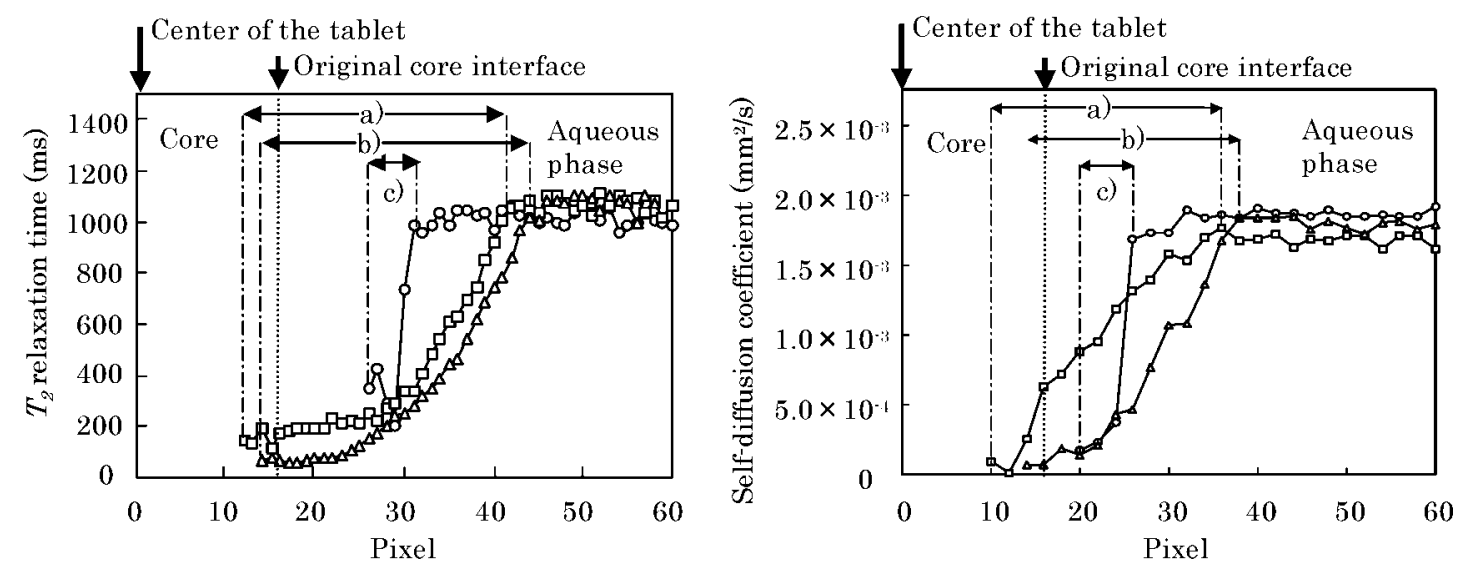

Fig. 2. One-Dimensional $T_{2}$ and ADC Maps of the LH41, HPMC and HPC Tablets after $20 \mathrm{~h}$ Hydration in Water in the Coronal Direction O: LH41, $\square:$ HPMC, $\triangle$ : HPC, a) Gel-layer (HPMC), b) Gel-layer (HPC), c) Gel-layer (LH41).

thin gel-layer may be located in a highly restricted environment, suggesting that a strong hydrogen-bonding interaction occurs between the absorbed water molecules and the hydroxyl groups of the LH41 polymer chains.

As shown in Fig. 2, similar tendencies were observed for hydrated LH41, HPMC, and HPC tablets in the one-dimensional ADC maps. The ADC values of the water in the gellayer were remarkably reduced in all hydrated tablets compared with those values in the outer aqueous phase. This result indicates that the water diffusivity is restricted in the gellayer. As described above, the $T_{2}$ and ADC maps can provide various kinds of information about the dimensional changes of water mobility and diffusivity during hydration and water mobility in the hydrated tablets.

Images of Various Tablets with Drug during Hydration Figure 3 shows typical $T_{2}$-weighted images taken through the coronal planes of the three kinds of tablets immersed in water for $20 \mathrm{~h}$. The growth of the gel-layer and the decrease in size of the glassy core can be clearly seen in the hydrated LH41/theophylline (THEO) tablets. Moreover, a deformation of the edge of the tablet was also observed during the swelling process. The same phenomena were observed in the hydrating LH41/procaine amide hydrochloride (PRAH) tablets; however, the extent of the gel-layer remarkably increased, because the procaine amide hydrochloride is more soluble compared with the theophylline. In the hydrating HPMC/THEO and HPC/THEO tablets, similar tendencies were observed. In the hydrating HPMC/PRAH and HPC/PRAH tablets, the gel-layers were expanded over into the glass bottle, whereas the glassy cores were perfectly faded away, at the same time.

One-Dimensional $T_{2}$ and ADC Maps of the Hydrating LH41 Tablets with Drug Figure 4 shows the one-dimensional $T_{2}$ and ADC maps across the LH41/procaine amide hydrochloride $(3: 1)$ tablets immersed in water for $20 \mathrm{~h}$. The moieties of the glassy core, gel-layer, and aqueous phase appear in the pixel ranges of 0 to 14,14 to 32 , and 32 to 60 , respectively, in the $T_{2}$ map. In the case of the tablet containing a drug, an increased amount of water was incorporated into the tablet, and a wider gel-layer was formed around the core interface compared with that formed for the tablet without drug. The $T_{2}$ and ADC values of the water in the wide gellayer were very short compared with those in the outer aque- (a) LH41/THEO

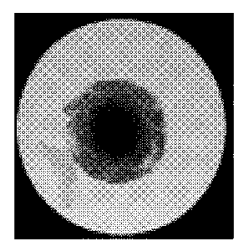

(b) HPMC/THEO

(c) HPC/THEO

(d) LH41/PRAH
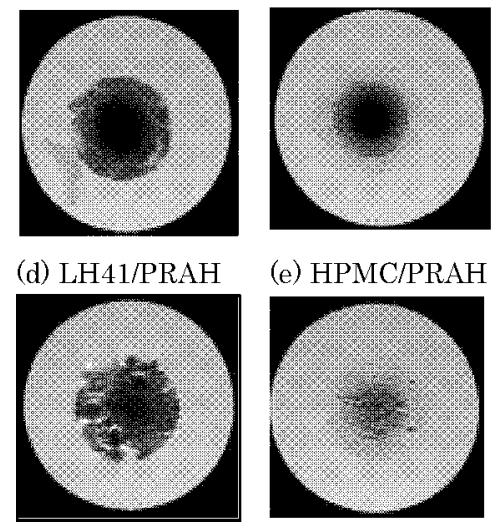

(e) HPMC/PRAH
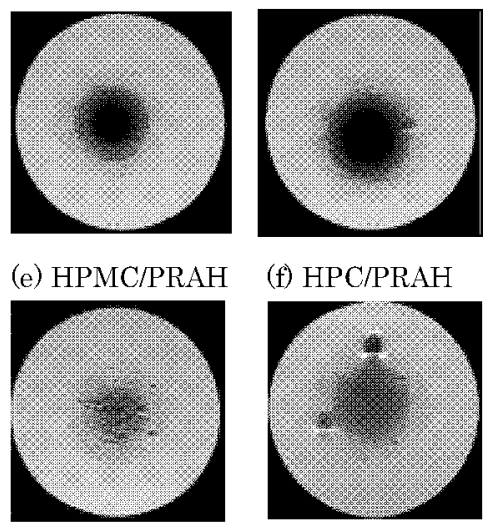

(f) HPC/PRAH

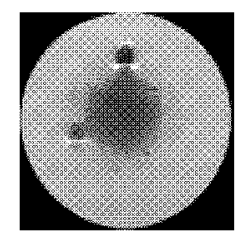

Fig. 3. Images of LH41, HPMC and HPC Tablets with Drugs Undergoing Hydrating after $20 \mathrm{~h}$ in the Coronal Plane

ous phase. These results indicate that the hydrated LH41 tablet containing a drug swelled remarkably and formed a wide gel-layer, and that the water in the wide gel-layer is located in a highly restricted environment.

Relationship between $\boldsymbol{T}_{\mathbf{2}}$ and Drug Release Rate Figure 5 shows a plot of the drug released from the tablets after $20 \mathrm{~h}$ as a function of a radial-averaged $T_{2}$. The $T_{2}$ was calculated using the average of signal intensity in the formed gellayer. In comparison of the tablets including the same drug, the release rate decreased as follows: LH41 $>$ HPMC $>$ HPC. The release rates also depended on the solubility of the drug. Furthermore, the tablets with polymer: drug ratio $(3: 1)$ provided a slower release compared with the tablets with the ratio $(2: 1)$. As shown in Fig. 5, there seemed to be a positive correlation between the drug release rate and the $T_{2}$ of ${ }^{1} \mathrm{H}$ proton in the gel-layer. This result suggests that measurement of ${ }^{1} \mathrm{H}$ proton mobility in the gel-layer is useful for evaluating the drug release from various hydrophilic matrices.

\section{Conclusions}

A localized analysis method for MRI was developed for mapping the water mobility and diffusivity in the hydrated matrix tablets used for controlled release drugs. One-dimensional $T_{2}$ and ADC maps can provide much information about dimensional changes in the tablets during hydration 

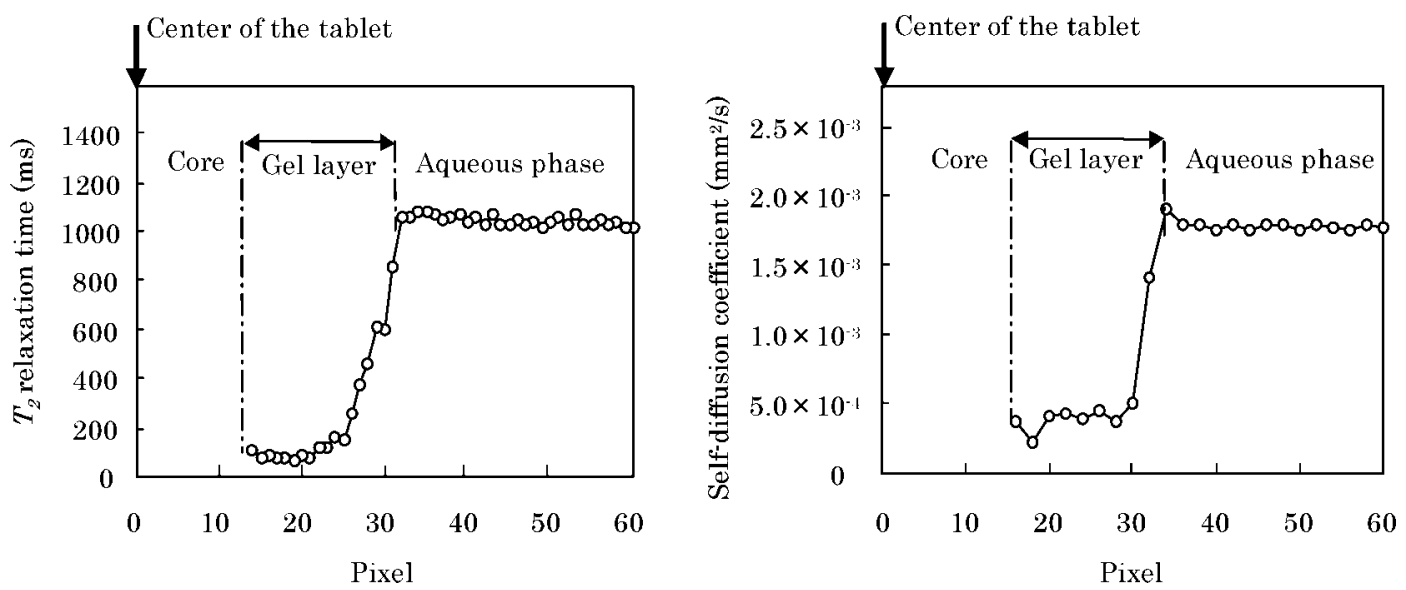

Fig. 4. One-Dimensional $T_{2}$ and ADC Maps of the LH41/PRAH Tablet after $20 \mathrm{~h}$ Hydration in Water in the Coronal Direction

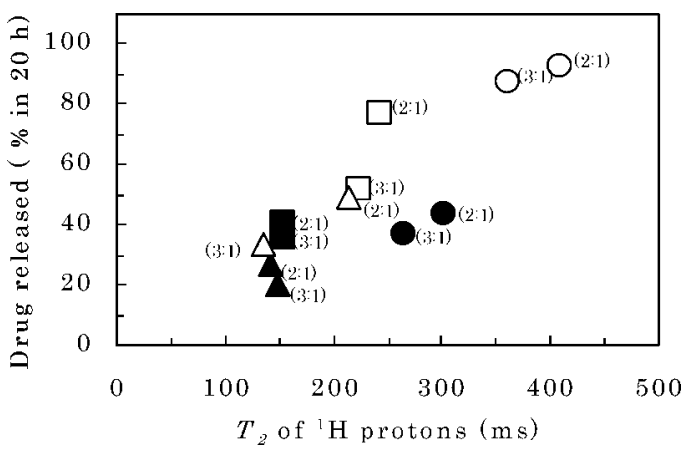

Fig. 5. The Relationship between the Amount of the Drug Released from Cellulose Matrix Tablets in $20 \mathrm{~h}$ and the $T_{2}$ of ${ }^{1} \mathrm{H}$ Protons in the Gel-layer of the Tablets Undergoing Hydration after $20 \mathrm{~h}$

-: LH41/THEO, O: LH41/PRAH, ם: HPMC/THEO, $\square$ : HPMC/PRAH, HPC/THEO, $\triangle$ : HPC/PRAH. ( ): polymer : drug ratio.

and the water mobility and diffusivity in the tablets. It was observed that the swelling of LH41 was much less than those of HPC and HPMC. Water mobility drastically decreased across the gel-layer of the hydrated LH41 tablet, suggesting that the water in the hydrated tablets of all three formulations is highly restricted. A correlation was observed between the $T_{2}$ of ${ }^{1} \mathrm{H}$ proton in the gel-layer of the tablets and the drug release rate from the tablets.

Acknowledgments This work was supported by a grant from The Japan Health Sciences Foundation. The authors thank Toyohiko Hirota and Kazuharu Aoyagi, Research Technology Center, Daiichi Pharmaceutical Co., Ltd., for their technical assistance.

\section{References}

1) A part of this study was presented at the 12th Academy of Pharmaceutical Science and Technology of Japan, Ohmiya, on April 1997.

2) Alderman D. A., Int. J. Pharm. Tech. and Prod. Mfr., 5, 1—9 (1984).

3) Nakano M., Ohmori N., Ogata A., Sugimoto K., Tobino Y., Iwaoku R., Juni K., J. Pharm. Sci., 72, 378-380 (1983).

4) Nakagami H., Nada M., Drug Design and Delivery, 7, 321-332 (1991).

5) Nakagami H., Nada M., Drug Design and Discovery, 8, 103-116 (1991).

6) Kawashima Y., Takeuchi H., Hino T., Niwa T., Lin T., Sekigawa F., Kawahara K., Pharm. Res., 10, 351-357 (1993).

7) Kojima M, Ando S., Kataoka K., Hirota. T., Aoyagi K., Nakagami H., Chem. Pharm. Bull., 46, 324-328 (1998).

8) Carr H. Y., Purcell E. M., Phys. Rev., 94, 630 - 638 (1954).

9) Stejskal E. O., Tanner J. E., J. Chem. Phys., 42, 288-292 (1965). 